\title{
OUTCOME OF ACUTE LIVER FAILURE DUE TO HEPATITIS A TREATED WITH MEDICAL MANAGEMENT
}

Thulaseedharan Nallaveettil Kesavan'1, Shajit Sadanand², Arun Thomas Edathumpadikal Thomas 3 , Bhagya Shaji ${ }^{4}$

1 Professor and HOD, Department of General Medicine, Calicut Medical College.

${ }^{2}$ Associate Professor, Department of General Medicine, Calicut Medical College.

3 Junior Resident, Department of General Medicine, Calicut Medical College.

4Junior Resident, Department of General Medicine, Calicut Medical College.

\section{ABSTRACT}

\section{BACKGROUND}

Acute liver failure is a heterogeneous entity and its prognosis varies with the aetiology. In India and other developing countries, hepatitis A virus is an important cause of acute liver failure. The prognostic factors and outcome of such patients should be studied separately.

\section{AIM OF THE STUDY}

To study the outcome of patients with acute liver failure due to hepatitis A treated with intensive supportive care and to determine the prognostic factors predicting the transplant free survival.

\section{MATERIALS AND METHODS}

In this observational study, all patients admitted in our hospital with ALF due to hepatitis A virus infection during the period of 3 years from January $1^{\text {st }} 2013$ to December $31^{\text {st }} 2015$ were selected; 40 patients satisfied the inclusion and exclusion criteria. Detailed history taking, physical examination, haematological and biochemical investigations were performed. The day-to-day progress and treatment given until discharge or death were recorded.

\section{RESULTS}

Overall mortality in acute liver failure due to hepatitis A was 30\%. Transplant free survival was $100 \%$ in patients with grade I and II encephalopathy, $66.6 \%$ in grade III encephalopathy and $22.2 \%$ in grade IV encephalopathy (P less than 0.001 ). Extrahepatic manifestations were observed in 29 patients (72.5\%), the most common was thrombocytopenia in 22 patients (55\%) followed by acute kidney injury in 12 patients (30\%).

\section{CONCLUSIONS}

The grade of hepatic encephalopathy was the single most important factor that determined the prognosis. Patients with grade I and II encephalopathy had 100\% spontaneous survival rate.

\section{KEYWORDS}

Acute Liver Failure, Hepatitis A, Liver Transplantation.

HOW TO CITE THIS ARTICLE: Kesavan TN, Sadanand S, Thomas ATE, et al. Outcome of acute liver failure due to hepatitis A treated with medical management. J. Evolution Med. Dent. Sci. 2016;5(17):834-837, DOI: 10.14260/jemds/2016/193

\section{INTRODUCTION}

Acute Liver Failure (ALF) is a potentially lethal syndrome defined by the occurrence of encephalopathy, coagulopathy and jaundice in an individual with a previously normal liver that triggers a cascade of events, leading to multiple organ failure and often death. In India, HAV and HEV are important causes of acute viral hepatitis and acute liver failure. ${ }^{[1-3]}$ There has been an increasing trend in the prevalence of HAV as aetiology of acute hepatitis and ALF. HAV infection is responsible for 10-30 percent of acute viral hepatitis and 5-15 percent of Acute Liver Failure (ALF) cases in India.[1-3]

Financial or Other, Competing Interest: None.

Submission 15-01-2016, Peer Review 11-02-2016,

Acceptance 16-02-2016, Published 27-02-2016.

Corresponding Author:

Thulaseedharan Nallaveettil Kesavan,

Professor and HOD, Department of General Medicine,

Calicut Medical College,

Kozhikode-673008,

Kerala, India.

E-mail: dr.thulaseedharan@gmail.com

DOI: 10.14260/jemds/2016/193
The treatment for acute HAV infection is largely supportive, but as many as $50 \%$ of HAV patients with Acute Liver Failure (ALF) may die or require emergency liver transplantation.[4-6] Early deaths in ALF are often caused by cerebral oedema or cardiovascular collapse, whereas late deaths tend to result from sepsis and multiple organ failure. Early identification of patients with a poor prognosis is important, but it is difficult due to the variability in patient survival and the unpredictability of subsequent complications. It appears that survival after acute liver failure is determined by multiple factors such as aetiology, grade of coma on admission, ability to regenerate a healthy liver and the absence of significant complications. The most widely applied prognostic system is the King's College Hospital Criteria $(\mathrm{KCH}$ Criteria), developed from a retrospective cohort of nearly 600 patients.[7] This criteria incorporates both the aetiology of ALF and clinical parameters of the disease. There are very few studies addressing the issues of acute liver failure according to a specific aetiology, which is indeed the most important prognostic factor in all the prognostic models. Even fewer studies are available regarding ALF due to HAV infection. 


\section{AIMS OF THE STUDY}

To study the outcome of patients with acute liver failure due to hepatitis A treated with intensive supportive care and to determine the prognostic factors predicting the transplant free survival.

\section{MATERIALS AND METHODS}

Our study was a hospital based observational study conducted in Calicut Medical College during the period of 3 years from January $1^{\text {st }} 2013$ to December $31^{\text {st }} 2015$. All patients above the age of 12 years with clinical features consistent with acute hepatitis A and a positive serology for IgM HAV who developed acute liver failure were included in the study. Patients with seropositivity for Hepatitis B, C and E, dengue virus or any other agent known to cause hepatitis, peripheral smear showing malarial parasite or positive rapid malarial antigen test and recent history of exposure to any hepatotoxic drugs or toxins were excluded from the study population. Acute liver failure was defined in our study as evidence of coagulation failure defined by International Normalized Ratio (INR) $\geq 1.5$ and any degree of mental alteration (Encephalopathy) in a patient with no underlying chronic liver disease.

Detailed history was obtained from all the patients and physical examination, complete blood counts, ESR, renal and liver function tests, serum electrolytes, PT, INR, ultrasonography of the abdomen, IgM HAV, IgM HEV, anti HCV, HBsAg, peripheral smear for malarial parasite and rapid malarial antigen test were done. All the patients were given intensive supportive care. Patients with deteriorating clinical course were given the option for liver transplantation. Those patients who opted for liver transplantation were not included in this study. The day-to-day progress and the treatment given were also recorded until discharge from the hospital or death.

\section{RESULTS}

Out of the 637 patients with hepatitis A who required inpatient care during the 3-year study period, 40 patients satisfied the inclusion and exclusion criteria of our study. 3 patients who opted for liver transplantation were excluded; 23 (57.5\%) were males and 17 (42.5\%) were females. Age of the patients varied from a minimum age of 14 years to a maximum age of 58 years. Mean age was 27.4 years; 9 patients were in the age group 12-19 years, 24 patients in the age group 20-39 years and 7 patients with age above 40 . Overall, mortality in patients treated with intensive supportive care was $30 \%$.

All the 40 patients studied had a history of fever with duration ranging from 1 day to 30 days and mean duration of 4.8 days. Only 9 patients had fever at the onset of hepatic encephalopathy. The interval between the onset of fever to the onset of encephalopathy ranged from a minimum of 3 days to a maximum of 30 days with a mean of 7.5 days. Either one or more of the classical symptoms of hepatitis (Anorexia, nausea and vomiting) were present in all the 40 patients.

Grade I hepatic encephalopathy was observed in 5 patients $(12.5 \%)$, grade II in 11 patients $(27.5 \%)$, grade III in 15 patients $(37.5 \%)$ and grade IV in 9 patients $(22.5 \%) ; 5$ patients $(12.5 \%)$ were anicteric at the onset of hepatic encephalopathy, defined by no clinical jaundice and total bilirubin level less than $3 \mathrm{mg} / \mathrm{dL}$. These patients later developed clinical jaundice. Prothrombin time and INR were analysed only at the onset of hepatic encephalopathy, as the majority of patients (95\%) had later received fresh frozen plasma; 27 patients had INR $<5,4$ patients had INR between 5 and 9 , and 9 patients had INR $>9$ at the onset. The lowest WBC count at the time of onset of encephalopathy was $3,500 / \mathrm{mm}^{3}$ and the highest was $23,800 / \mathrm{mm}^{3}$ with a mean of $9,400 / \mathrm{mm}^{3}$.

Extrahepatic manifestations were observed in 29 patients (72.5\%), of which the most common was thrombocytopenia (Defined as platelet count less than 1.5 lakh) in 22 patients. Others were acute kidney injury in 12 patients, seizures in 4 patients and arthritis in 1 patient. Out of the 22 patients with thrombocytopenia, 3 patients had platelet count less than $50,000 / \mathrm{mm}^{3}$. Out of the 12 patients with acute kidney injury, 6 patients had KDIGO stage III AKI, of which 3 patients died.

All patients received gut sterilisation with Rifaximin, bowel wash and parenteral antibiotics (Piperacillin/ Tazobactam combination or Cefotaxime).

Except for 2 patients, all others were given prophylactic fresh frozen plasma. Out of the 40 patients, 12 patients (30\%) succumbed to their illness. All the 12 patients who died had either grade III or IV encephalopathy. Clinically evident life threatening bleeding (Upper GI bleed) was present in one patient.

Severe grades of hepatic encephalopathy as defined by grade III and IV were more frequently observed in older age groups. Only $33.3 \%$ of patients in age group $12-19$ years had grade III and IV encephalopathy when compared to $71.2 \%$ in patients with age $>40$ years, but this difference was not statistically significant. Higher grades of encephalopathy (Grade III and IV) were more frequent in females $(76.5 \%$ vs $47.2 \%$ ), but this observation was not statistically significant.

Acute kidney injury was more frequently observed in higher age groups; 6 out of the 7 patients older than 40 years had acute kidney injury when compared with 5 out of 19 in age group 20-39 years and 1 out of 9 in patients younger than 20 years ( $p=0.002)$. The mean duration of hospital stay was 9 days in patients younger than 20 years, 11 days in age group 20-39 years and 20 days in patients older than 40 years $(p=0.008)$. Higher death rate was observed among patients in older age groups (22.2\% in age less than 20 years, $29 \%$ in age $20-39$ years and $42.9 \%$ in age above 40 years), but this observation was not statistically significant.

Mortality rate was $77.8 \%$ in grade IV encephalopathy, $33.3 \%$ in grade III encephalopathy and $0 \%$ in grade I and II encephalopathy (Table 1). Association between mortality and grade of encephalopathy was statistically significant (P less than 0.001). Duration of fever, fever to encephalopathy time interval and presence of fever at the onset of encephalopathy did not have statistically significant association with severity of hepatic encephalopathy, extrahepatic manifestations or survival.

Total WBC count, bilirubin level, INR, serum albumin and peak SGPT measured at the onset of encephalopathy did not have any statistically significant association with the severity of hepatic encephalopathy or survival. The magnitude of maximum fall of SGPT (Absolute and percentage) in 24 hours also did not have any statistically significant association with the severity of hepatic encephalopathy or survival.

\section{DISCUSSION}

Spontaneous survival rate in acute liver failure due to hepatitis A in our study was $70 \%$. Study by Schiodt FV, et al. on viral 
hepatitis related ALF in US also reported 69\% spontaneous survival rate in cases with HAV as the aetiology.[4] The grade of hepatic encephalopathy was the single most important factor that decided the prognosis. There was $100 \%$ transplant free survival in grade I and II encephalopathy, whereas transplant free survival was $66.6 \%$ in grade III encephalopathy and $22.2 \%$ in grade IV encephalopathy. ALF due to hepatitis A virus needs separate transplantation recommendations as these patients have a better outcome when compared with ALF due to other aetiologies. ${ }^{4}$ Liver transplantation is not required in patients with grade I and II encephalopathy as these patients have very good prognosis, $100 \%$ in our study.

Transplant free survival for patients with grade IV encephalopathy is very poor; hence, they should undergo liver transplantation with minimum waiting time. +The patients with grade III hepatic encephalopathy have a transplant free survival (66.6\% in our study) comparable to the success rates reported from various high volume living donor liver transplantation centres (1 year post-transplant survival rate 59-88\%).[8-12]

The estimated risk of mortality and morbidity currently associated with live donor hepatectomy is $0.4 \%$ and $35 \%$, respectively.[13] These two factors need to be considered while making treatment decisions. Advanced age and the presence of acute kidney injury were other factors associated with poor outcome in our study. No other clinical or biochemical parameters including the magnitude of maximum fall in SGPT (Absolute and percentage) in 24 hours, had any statistically significant association with the outcome of the patients.

Thrombocytopenia (55\% patients) and acute kidney injury $(30 \%$ patients) were the common extrahepatic manifestations observed in our study. Patients with KDIGO stage III AKI had 50\% mortality. Many factors such as volume depletion, systemic hypoperfusion, co-existing sepsis, immune mediated glomerulonephritis and hepatorenal syndrome are responsible for acute kidney injury in this setting. ${ }^{[14]} \mathrm{AKI}$ complicates ALF in 40 to $50 \%$ of cases and is significantly associated with a poor prognosis in various other studies.[7,15,16]

Theoretically, prophylactic treatment with FFP in the absence of bleeding is not advised for two reasons; first, the decrease in prothrombin time after FFP administration decreases the accuracy with which prognosis can be judged and second, it results in volume overload and increased ICP. $[17,18]$ In clinical practice, FFP may be beneficial, especially when options for liver transplantation are not available due to various reasons. In our study, $95 \%$ of patients received prophylactic FFP. Only one patient developed major lifethreatening bleed.

\section{CONCLUSIONS}

The grade of hepatic encephalopathy is the single most important factor that decides the prognosis. Other predictors are age and the presence of acute kidney injury. Patients with grade I and II encephalopathy have excellent prognosis with conservative management. Prognosis of patients with grade IV hepatic encephalopathy is poor and they should undergo immediate liver transplantation.

The patients with grade III hepatic encephalopathy have a transplant free survival comparable to the success rates after transplantation as reported from various high volume living donor liver transplantation centres, hence treatment decisions should be individualised. All patients on conservative management may benefit from prophylactic fresh frozen plasma to decrease major bleeding.

\begin{tabular}{|c|c|}
\hline Grade of Hepatic Encephalopathy & Mortality \\
\hline Grade I (n=5) & $0 \%$ \\
\hline Grade II (n=11) & $0 \%$ \\
\hline Grade III (n=15) & $33.3 \%(\mathrm{n}=5)$ \\
\hline Grade IV (n=9) & $77.8 \%(\mathrm{n}=7)$ \\
\hline \multicolumn{2}{|c|}{ Table 1 } \\
\hline
\end{tabular}

\section{REFERENCES}

1. Thayumanavan L, Jegannathan ASA, Krishnamurthy, et al. Profile of hepatitis A-E in sporadic acute virus hepatitis in Madurai (Abstract). Indian J Gastroenterol 2003;22 (Suppl 1):A91.

2. Arora NK, Nanda SK, Gulati S, et al. Acute viral hepatitis types E, A and B singly and in combination in acute liver failure in children in north India. J Med Virol 1996;48:21521.

3. Viral hepatitis in India, 2010 to 2013. NCDC Newslett 2014;3:12.

4. Schiodt FV, Davern TJ, Shakil AO, et al. Viral hepatitisrelated acute liver failure. Am J Gastroenterol 2003;98:448-453.

5. Schiodt FV, Atillasoy E, Shakil AO, et al. Etiology and outcome for 295 patients with acute liver failure in the United States. Liver Transpl Surg 1999;5:29-34.

6. Rezende G, Roque-Afonso AM, Samuel D, et al. Viral and clinical factors associated with the fulminant course of hepatitis A infection. Hepatology 2003;38:613-618.

7. O'Grady JG, Alexander GJM, Hayllar KM, et al. Early indicators of prognosis in fulminant hepatic failure. Gastroenterology 1989;97:439-455.

8. Yamashiki N, Sugawara Y, Tamura S, et al. Outcomes after living donor liver transplantation for acute liver failure in Japan: results of a nationwide survey. Liver Transpl 2012;18:1069-1077.

9. Uemoto S, Inomata Y, Sakurai T, et al. Living donor liver transplantation for fulminant hepatic failure. Transplantation 2000;70:152-157.

10. Ikegami T, Taketomi A, Soejima Y, et al. Living donor liver transplantation for acute liver failure: a 10-year experience in a single center. J Am Coll Surg 2008;206:412-418.

11. Liu CL, Fan ST, Lo CM, et al. Right-lobe live donor liver transplantation improves survival of patients with acute liver failure. Br J Surg 2002;89:317-322.

12. Campsen J, Blei AT, Emond JC, et al. Adult-to-Adult living donor liver transplantation cohort study group. Outcomes of living donor liver transplantation for acute liver failure: the adult-to-adult living donor liver transplantation cohort study. Liver Transpl 2008;14:1273-1280.

13. Barr ML, Belghiti J, Villamil FG, et al. A report of the Vancouver Forum on the care of the live organ donor: lung, liver, pancreas and intestine data and medical guidelines. Transplantation 2006;81:1373-1385.

14. Bihari DJ, Gimson AE, Williams R. Cardiovascular, pulmonary and renal complications of fulminant hepatic failure. Semin Liver Dis 1986;6:119-128. 
15. Ring-Larsen H, Palazzo U. Renal failure in fulminant hepatic failure and terminal cirrhosis: a comparison between incidence, types and prognosis. Gut 1981;22:585591.

16. Jain S, Pendyala P, Varma S, et al. Effect of renal dysfunction in fulminant hepatic failure. Trop Gastroenterol 2000; 21:118-120.
17. Polson J, Lee WM. American association for the study of liver disease. AASLD position paper: the management of acute liver failure. Hepatology 2005;41:1179-1197.

18. Gazzard BG, Henderson JM, Williams R. Early changes in coagulation following a paracetamol overdose and a controlled trial of fresh frozen plasma therapy. Gut 1975;16:617-620. 